\title{
Padova Emotional Dataset of Facial Expressions (PEDFE): a unique dataset of genuine and posed emotional facial expressions
}

\author{
A.Miolla · M.Cardaioli · C.Scarpazza
}

the date of receipt and acceptance should be inserted later

\begin{abstract}
Facial expressions are among the most powerful signals for human beings to convey their emotional states. Indeed, emotional facial datasets represent the most effective and controlled method of examining humans' interpretation of and reaction to various emotions. However, the scientific research on emotion mainly relied on static pictures of facial expressions posed (i.e., simulated) by actors, creating a significant bias in emotion literature. This dataset tries to fill this gap, providing a considerable amount $(N=1458)$ of dynamic genuine $(N=707)$ and posed $(N=751)$ clips of the six universal emotions from 56 participants. Furthermore, pictures displaying frame by frame the temporal dynamic of the expression, are also available for each clip. Notably, all stimuli were validated by 122 human observers. Hit rates for emotion and genuineness, as well as the mean, standard deviation of genuineness, and intensity perception, are provided for each clip.
\end{abstract}

Keywords facial expressions · genuine emotions · posed emotions · emotion dataset

\section{Introduction}

Facial expressions represent an innate and automatic behavior component of emotional and social communication (Darwin, 1872, Jack et al., 2016, Motley and Camden, 1988, Zloteanu et al., 2018). Emotional facial expressions, in particular, have

\section{A.Miolla}

Department of General Psychology, University of Padua, Italy

E-mail: alessio.miolla@phd.unipd.it

M.Cardaioli

Department of Mathematics, University of Padua, Italy

GFT Italy, Milan, Italy

E-mail: matteo.cardaioli@phd.unipd.it

C.Scarpazza

Department of General Psychology, University of Padua, Italy

E-mail: cristina.scarpazza@gmail.com 
a communicatory function that conveys specific information to the receiver $\overline{\mathrm{An}}-$ drew, 1963; Darwin and Prodger, 1998; Ekman et al., 1969; Jack et al., 2012, Jack and Schyns, 2015). For example, an expression of happiness through a smile in response to a particular behavior, increases the probability that the action will be repeated in the future, differently from an angry or sad face (Motley and Camden. 1988). In this sense, the nature and the interpersonal function of the emotional facial expressions signal a feeling (or an intention) that predicts different social outcomes (Darwin, 1872, Ekman, 1972). It is precisely for this reason that knowing what another person feels, accurately deciphering what someone is trying to communicate, is extremely important in day-to-day social interactions (Johnston et al. 2010). However, the great variability of facial expressions makes this task very hard. In fact, emotions conveyed by faces can change under several parameters. We can display different varieties of expressions: some intense and sustained, while others are subtle and fleeting (Ambadar et al. 2005). One of the most high level and critical communication features is related to the perception of authenticity of the emotion expressed (Lu et al., 2020, Rooney et al., 2012). In fact, we can express emotions spontaneously, triggered by real circumstances (i.e., "eventelicited") Dawel et al. (2017). For example, someone might be scared because he is genuinely afraid of a snake or be sad because of the loss of a loved one. Conversely, we can deliberately feign or pose emotions in the absence of a congruent underlying context in order to receive adaptive advantages. These expressions reflect the strategic intent of the sender in the absence of felt emotions (Ekman and Rosenberg, 2005).

For example, pretending to be sad can be a useful strategy to take advantage of a perceiver's reciprocal kindness or compensatory behavior in response (Reed and DeScioli, 2017). The endogenous nature of emotional experiences (i.e., genuine or posed) completely changes the observer's perception and reaction. In social interactions, perceiving others' emotional reactions as genuine might promote social interaction and increase the expresser's trustworthiness (Reed and DeScioli, 2017). For example, Johnston et al. (2010) showed how genuine (or spontaneous) smiles make perceivers more cooperative than posed smiles. In psychotherapy, therapists' genuineness, authenticity, and honesty promote to enhance their credibility, which is essential for promoting therapeutic alliance and patients' trust (Dowell and Berman, 2013, Jung et al., 2015, Lu et al., 2020, Schnellbacher and Leijssen, 2009). Furthermore, in movies, the perception of realism in the actor's performance may promote a more emphatic mechanism and a more emotional contagion of the perceivers (Rooney et al., 2012). From a neuropsychological point of view, it has also been argued that genuine and fake emotions may recruit different components of emotional contagion (Manera et al., 2013). For example, there is evidence that genuine smiles are associated with the experience and physiological activations of positive emotions, while faked ones with the experience and physiological activation of negative emotions (Davidson et al. 1990, Ekman et al., 1990, Soussignan, 2002 ).

Despite this evidence, to the best of our knowledge, only two recent studies used spontaneous facial expressions so far (Künecke et al., 2017, Vergallito et al., 2020). Virtually all the previous research investigating facial expressions have focused on posed (or fake) emotions (Dawel et al. 2017, Tcherkassof et al., 2013), raising serious doubts about the ecological impact of these stimuli (Barrett et al., 2019; Russell, 1994; Tcherkassof et al., 2013, Wallbott, 1990; Wallbott 
and Scherer, 1986, Zuckerman et al., 1976). Spontaneous/genuine and posed/fake emotional expressions differ in their temporal and morphological characteristics, such as duration, intensity, and asymmetry (Cohn and Schmidt, 2003, Ekman, 1997, Sato and Yoshikawa, 2004; Valstar and Pantic, 2010; Wehrle et al., 2000, Yoshikawa and Sato, 2006). Indeed, posed emotions display stereotypical and exaggerate facial configuration that is rarely met in real life (Barrett et al., 2019). On the other side, spontaneous emotions in real life are usually less intense, more subtle, and more difficult to detect (Dawel et al., 2017; Tcherkassof et al., 2013). As a result of the strict focus on prototypical posed facial expressions, it is evident that researchers may have underestimated the considerable differences between spontaneous and posed emotional facial expressions. It is thus still not known whether our knowledge of processing of emotions conveyed by faces is biased by the fact that studies have been conducted using stimuli displaying stereotypical emotions. This important bias makes unknown whether the results on emotions perception from faces so far available within the scientific literature are driven by the (un)conscious perception of the non authenticity of the perceived emotions. Even more importantly, it is not known whether results obtained using posed emotions are generalizable to genuine emotions.

These research questions are still unanswered also because the scientific community is still devoid of a validated dataset of stimuli including both genuine and posed emotions from the same actors. Although some datasets including genuine and posed emotions seem to be present in literature (please see Krumhuber et al. (2017) for a review), their usefulness is limited as the emotions expressed are not genuine as elicited by methods that limited the spontaneity of the subjects' facial displays (e.g., subjects were aware of the aim of the studies, thus creating a barrier in the elicitation of spontaneous emotions) (Cheng et al., 2018, Kulkarni et al., 2018; Novello et al., 2018). In addition, some of them are not validated (Cheng et al., 2018; Kulkarni et al., 2018), or they are displayed only through static pictures (Dawel et al., 2017; Novello et al., 2018).

The current work aims to enrich the future research of emotions providing the scientific community with a new dataset of emotional stimuli conveyed by faces, that includes a considerable amount of both spontaneous and posed emotional facial expressions of the six basic emotions. We called this dataset Padova Emotional Dataset of Facial Expressions (PEDFE). The contributions of the current research are multiple: first, PEDFE includes a considerable amount of emotional clips for both spontaneous and posed emotions. The same emotion is displayed genuinely and posed for each participant, allowing a direct comparison (i.e., intra-subject and between-subject) between these two ways to express the emotional facial expressions. Second, the elicitation protocol uses a multimodal sensorial perception to elicit emotions as natural as possible, avoiding any restrictions or influences by the researcher (please see the paragraph "Methods"). To the best of our knowledge, the current emotion elicitation protocol has more tasks (i.e., 15) than the other reported methods. Third, all stimuli were validated by asking subjects to rate each clip according to the emotion, genuineness, and intensity of the facial expression perceived. It implies an essential step in creating emotional datasets that most of the datasets displaying genuine and posed emotions neglected. Last, PEDFE qualifies as the first spontaneous dataset displaying only the face, removing all distracting variables from the background (e.g., hair, clothes, color of the 
background, ecc), and providing several advantages in research (Davies et al., 1994 . Minami et al., 2018; Tsao and Livingstone, 2008; Xu et al., 2017).

\section{Dataset Creation}

2.1 Participants selection procedure and compliance with ethical standards

Fifty-seven participants, aged between 20 and 30 years, took part in the experiment. Participants were randomly assigned to one of the two settings (please see Section 2.2). The sample was enrolled using an advertisement on the University Website and were compensated for their participation. Participants signed an informed consent before the beginning of the experiments. After reading this informed consent, they were still unaware of the purpose of the study and were unaware of being filmed. The participants were informed that they had the right to quit the experiment and withdrew their consent at any time. At the end of the session, participants were debriefed, and the study's real aims were revealed. They were also told they were recorded. One participant withdrew her consent, and her clips were permanently removed from the database. The experimental procedure and the emotional elicitation protocol submitted to the participants and described in the following paragraphs were approved by the Ethics Committee of the University of Padua (Protocol number: 2917). The participants' video recordings were included in the database only after they signed a written consent to use their videos for research purposes.

\subsection{Experiment setup}

The aim of the experimental procedure was to record spontaneous (i.e. stimulus elicited) emotions of participants while they watched emotional video or were performing simple tasks. For this reason, participants were left alone in an experimental room to decrease the possibility that embarrassment and social inhibition could affect the spontaneity of expressed emotion, impacting on the overt manifestation of emotions. The doors and windows were kept shut during the entire protocol to avoid external interference and allow participants a more in-depth emotional excursion during the tasks. Participants were set about one meter in front of a Lenovo ThinkPad T490. As it is known that awareness of the experimental aim can interfere with the spontaneity of overt emotional expression (Happy et al. 2015. Sebe et al., 2007), participants were unaware of the purpose of the experiment. For this reason, a cover story was created. In particular, participants were told they have to rate emotional valence of the videos, as already did for a previous study (Happy et al., 2015). They were also told that, in order to accurately assess emotions, they had to try to get immersed in the viewing experience and feel free to experience their emotions. Moreover, subjects were allowed to sit at their ease without any other restrictions inside the experimental room to avoid possible suspects or limit the emotions' naturalness.

The same protocol was submitted in one of the two following modalities in order to enrich the database with different viewing angles. The first setting was created based on the well-known assumption that awareness of being filmed might impacts 
on spontaneity of overtly expressed emotions. Thus, in this first setting, a hidden camera placed at the right room's top angle was used. Participants were thus totally unaware of being recorded, preserving the emotional reactions' spontaneity. The clips were recorded with a AW-HE40HWEJ-Panasonic at a distance of at least 2 meters, with an angular size of $20^{\circ}$, varying in accordance with the head movements of subjects. The second setting was thought with the aim to create video depicting the participants on a frontal view. For this reason, in the second setting, a Logitech C920 HD Pro Webcam, Full HD 1080p/30fps, was placed at the top of the computer screen used for the tasks. In this setting, to preserve the subjects' expressions' spontaneity, participants were told that the recording was necessary to study the eye movements and pupil dilatation while performing the valence rating task. The two experimental setups guarantee more options to the experimenter who will use the emotional stimuli by having the same emotions (both spontaneous and posed) with a front and a lateral view (see Fig. 1).

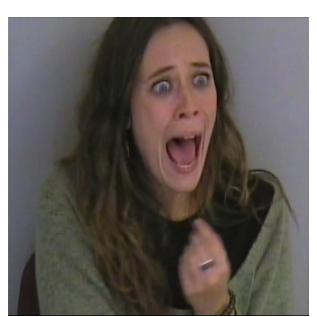

(a) First setting

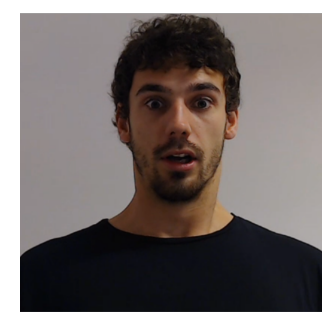

(b) Second setting

Fig. 1: Examples of fear expressions for the two settings.

\subsection{Emotion elicitation procedure}

Spontaneous emotional reactions were elicited with a multimodal protocol. Emotions were mostly triggered by watching emotion-inducing videos, which resulted to be the most effective stimuli for evoking emotional responses (Carvalho et al. 2012). The clips were selected from different stimuli that have been used for similar studies (Rottenberg et al. 2007), and from other sources such as international films, commercial spots, and YouTube clips. The length of the clips did not exceed 5 minutes according to the recommended size of the emotional video (Rottenberg et al., 2007). The emotions were not only elicited through passive elicitation by watching emotion-inducing videos. For example, anger was also triggered by using a rage game, well-tested stimuli to provoke anger, in which the emotion was elicited as a result of the encoder actively engaging with the game (Sneddon et al., 2011). Indeed, the typology of these games was designed to make the task very difficult to purposely increase a high level of frustration and anger to the players. As, in pilots trails, we found that anger is often repressed, we provide participants with a desktop punching ball. Finally, as olfactory stimuli can reliably elicit disgust and have been resulted in very efficiently in previous studies (Hayes et al. 2009a b; Zhang et al., 2016), an unpleasant odor was presented to the subject to induce a disgusting feeling. 
The spontaneous emotion elicitation protocol is summarized in Table 1 . Notably, more stimuli were chosen per emotion to enhance the probability of eliciting the target emotion and collecting more samples of clips displaying the same emotion for each subject. For example, in sadness, we used five tasks to trigger and collect sad facial expressions. This choice was due to the peculiar characteristics of sadness, which is associated with loss of muscular tone and a focus on inner thoughts and feelings (Ekman and Friesen, 2003, Izard, 1991) that make sadness more difficult to detect. The number of tasks used to elicit sadness as well as in other emotions and, in general, the size of the multi-modal elicitation protocol was thus extended to increase the chances to stimulate and collect more emotional facial expressions as possible from the same participant. The order of tasks from 1 to 14 was randomized across the subjects.

After the end of each task, participants were asked to identify the emotion they experienced within the six basic emotion and neutral. They were also given the possibility to report if they felt an emotion that was not included within the six basic ones. Furthermore, besides identifying the emotion felt, they were also asked to rate how much the emotion they felt was genuine on a Likert scale ranging from -7 to +7 where -7 corresponded to "completely not genuine" and +7 corresponded to "completely genuine", according with previous literature (Dawel et al., 2017). Finally, participants rated the intensity of the emotions experienced during the tasks on a scale ranging from 0 (None) to 9 (Strong) (Dawel et al., 2017). When the multimodal emotion elicitation protocol was successfully concluded, participants were asked to pose the six basic emotions multiple times, modulating the intensity of the posed emotions.

\subsection{Video extraction}

One of the authors (AM), a certified Facial Action Coding System (FACS) coder, extracted the facial expression of emotions present in the recorded videos. The clips' selection was made considering both the FACS's criteria (Ekman et al. 1978) and participants' self-reports.

FACS is a widely used protocol for recognizing and labeling all visually discernible facial movements, called Action Units (AUs). In addition, the manual proposes a list of possible combinations of AUs which are typically associated with emotions expressions (Ekman et al. 2002). The current method was used to reliably and accurately extract the emotional facial expressions shown by participants.

\footnotetext{
1 https://www . youtube. com/watch?v=URGUQlcAoNUab_channel=larablacklady

2 https://www.youtube.com/watch?v=F2bk_9T482g\&ab_channel=xXJEashXx

3 https://www.youtube.com/watch?v=cLCE9_JHjPE\&ab_channel=Mercating

4 https://www.youtube.com/watch?v=JHXObtJYcyI\&ab_channel=PhilBeastallFilms

5 https://www.youtube.com/watch?v=4_B6wQMd2eI\&ab_channel=WIACZO

6 https://www.youtube.com/watch?v=0grANlx7y2E\&ab_channel=PhillipNorthfield

7 https://www.youtube.com/watch?v=v3iPrBrGSJM\&ab_channel=Quirkology

8 https://flappybird.io/

9 https://www.gioco.it/gioco/scary-maze

10 https://www.youtube.com/watch?v=beAxdoCFnhw\&ab_channel= COMPILATIONPOPPINGVIDEOS
} 
Table 1: Multimodal protocol for Spontaneous and Posed emotion elicitation. Tasks are presented in this table in the same order they were presented to participants.

\begin{tabular}{|c|c|c|c|c|}
\hline Task & Emotion & Activity & Description & Lenght \\
\hline T1 & Sadness & $\begin{array}{l}\text { Watch a VIDEO: Death } \\
\text { of Mufasa, from the Lion } \\
\text { King } 1\end{array}$ & $\begin{array}{l}\text { The clip displayed the saddest part of } \\
\text { the movie, when Mufasa dies because } \\
\text { of Scar, and the touching reaction of } \\
\text { Simba. }\end{array}$ & $02: 42 \mathrm{~min}$ \\
\hline T2 & Sadness & Disney Pixar U $\mathrm{H}^{2}$ & $\begin{array}{l}\text { The scene where Ellie and Carl are } \\
\text { shown. Their relationship is being } \\
\text { shown as time passes from their wed- } \\
\text { ding to Ellie's death. }\end{array}$ & $04: 21 \mathrm{~min}$ \\
\hline T3 & Sadness & $\begin{array}{l}\text { "Giving without expecting } \\
\text { anything in return is the } \\
\text { best communication" } 3\end{array}$ & $\begin{array}{l}\text { Spot for Telecom in Thailand. The } \\
\text { story is about kindness rewarded over } \\
\text { the course of } 30 \text { years. }\end{array}$ & 03:08 $\min$ \\
\hline T4 & Sadness & "Love is a gift" & $\begin{array}{l}\text { It's a short film about a man counting } \\
\text { down the days to Christmas so he can } \\
\text { continue his yearly tradition sparked } \\
\text { by a tragic moment from the past. }\end{array}$ & $02: 25 \mathrm{~min}$ \\
\hline T5 & Sadness & $\begin{array}{l}\text { "Edeka } 2015 \text { Christmas } \\
\text { Commercial": }\end{array}$ & $\begin{array}{l}\text { Edeka's holiday commercial reminds } \\
\text { people of the important things in life } \\
\text { in a tragic piece of storytelling. }\end{array}$ & 01:30 min \\
\hline T6 & Surprise & The Invisible Gorilla ${ }^{6}$ & An experiment in Change Blindness. & 01:00 min \\
\hline $\mathrm{T} 7$ & Happiness & When Harry met Sally & $\begin{array}{l}\text { This is a classic and funny part to a } \\
\text { very good movie. The restaurant/deli } \\
\text { scene where Sally fakes an orgasm to } \\
\text { prove a point. }\end{array}$ & 02:46 min \\
\hline T8 & Surprise & $\begin{array}{l}\text { Colour } \\
\text { Trich } 7\end{array}$ & An experiment in Change Blindness. & 02:43 min \\
\hline T9 & Anger & Flappy Bir 8 & $\begin{array}{l}\text { A so-called "Rage game", namely a } \\
\text { game while gaming and can't accom- } \\
\text { plish your goal whatever that is, and } \\
\text { you get random from your lack of suc- } \\
\text { cess. }\end{array}$ & 05:00 min \\
\hline T10 & Fear & Scare Jumr ${ }^{9}$ & $\begin{array}{l}\text { A so-called jump scare, namely a game } \\
\text { intended to scare the audience by sur- } \\
\text { prising them with an abrupt change in } \\
\text { image, co-occurring with a frightening } \\
\text { sound. }\end{array}$ & 04:00 min \\
\hline T11 & Anger & Abused dog in a metro & $\begin{array}{l}\text { The clip showed the abuse of a dog, } \\
\text { beaten by his owner on a public metro. }\end{array}$ & 03:00 min \\
\hline T12 & Fear & Scare jump horror clip & $\begin{array}{l}\text { A classic horror clip aimed to scare } \\
\text { participants with frightening scenes } \\
\text { and spectral sounds. }\end{array}$ & $02: 28 \mathrm{~min}$ \\
\hline T13 & Disgust & Pimples squeezing ${ }^{10}$ & $\begin{array}{l}\text { Disgusting huge and ingrown pimples } \\
\text { are squeezed in the clip. }\end{array}$ & 05:00 min \\
\hline T14 & Disgust & Stinky potion & $\begin{array}{l}\text { A solution characterized by an un- } \\
\text { pleasant smell that causes a strong re- } \\
\text { action of disgust. }\end{array}$ & 01:00 min \\
\hline T15 & - & Simulation Session & $\begin{array}{l}\text { Participants were asked to pose each } \\
\text { emotion as authentic as possible for } 30 \\
\text { seconds each, trying to change their in- } \\
\text { tensity. }\end{array}$ & 06:00 min \\
\hline
\end{tabular}

In other words, the clips were selected only if the emotional expression (e.g., happiness) matched FACS criteria (e.g., AU6+12) and participants' self-report (e.g., they declare to have experienced happiness with a high level of genuineness). In the case in which multiple emotions were induced (e.g., happiness and surprise), the emotional facial expressions associated were both selected if the self-reports and facial changes of participants were in accordance with the emotions elicited. Conversely, if participants reported having felt constrained and not natural in the emotional experience (e.g., a score of -4 on the genuineness scale), all the expressions associated with the task were removed. Likewise, if participants showed a facial expression associated with an emotion (e.g., a scowl that may reflect anger), the facial change was not selected if participants did not report to have experi- 
enced anger. In fact, a scowl is not always a cue of anger but could instead reflect confusion or concentration. This strict procedure aims to reduce the selection of facial expressions that do not convey authentic and spontaneous emotions. Each clip was cut from the onset point (i.e., the first frame when the expression is visible) to the apex (i.e., the period during which the movement was held at the highest intensity reached) of the emotion. Additionally, if the same emotion(s) was repeatedly elicited in a task, the related expressions were selected multiple times as much as the number of times participants spontaneously expressed the emotion(s) reported, in order to increase the number of clips included in the final dataset and provide more trials of the same emotion for each participant. Lightworks (https://www.lwks.com/), a non-linear editing system (NLE) for editing and mastering digital video, was used to extract the emotional clips' perfect range frame.

\section{Results}

PEDFE contains clips and static pictures of 56 participants, displaying subtle to full-blown elicitation of different emotions. Overall, the number of emotional clips is 1731 (the exact number clips for each emotion and category are provided in (see Fig. 2), whose duration varies from 0.1 seconds(s) to 23.5 seconds(s).

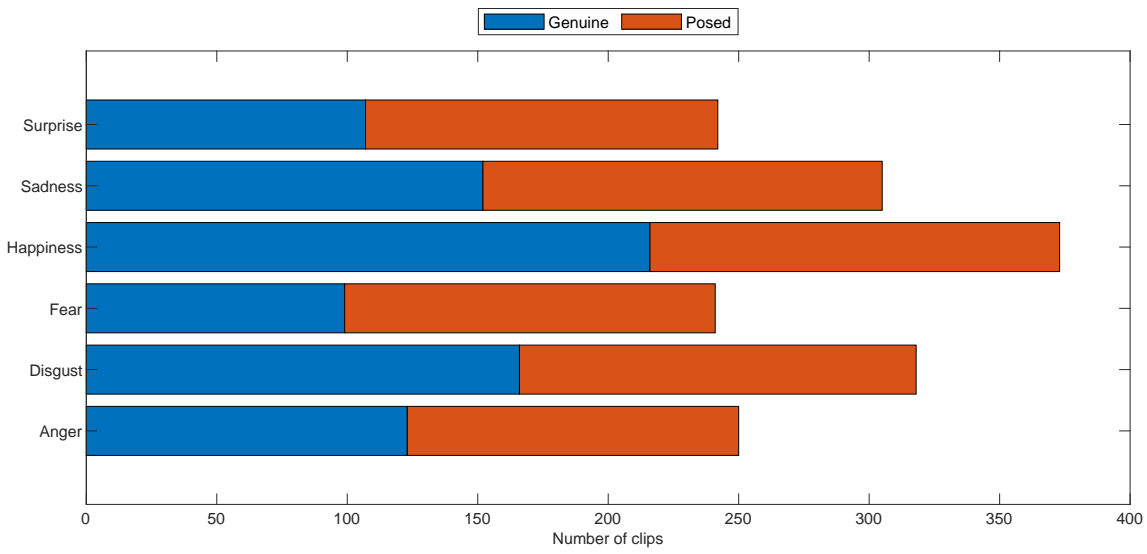

Fig. 2: Number of clips before the Validation, divided for emotion and type.

More precisely, the duration of the facial expressions varied in accordance with the emotion displayed. For example, sad clips last longer ( $M=5.35 \mathrm{~s}$; $S D=2.92 s)$ than other emotions such as happiness $(M=2.89 s ; S D=1.25 s)$, disgust $(M=2.81 s ; S D=1.33 s)$ or anger $(M=2.92 ; S D=1.38)$ because of the gradual evolution of sadness over a longer time-frame. Conversely, emotions like surprise $(M=1.94 s ; S D=1.04 s)$ or fear $(M=1.86 s ; S D=0.92 s)$ emerged and disappeared faster, lasting a few seconds at the most(Ekman and Friesen, 2003) The considerable amount of clips (i.e., 1731), as well as the self-reports given by participants, revealed the effectiveness of the elicitation protocol (please see Fig. 3 . 
Fig. 4). In fact, most participants reported, on average, to have experienced the emotion that the elicitation tasks aim to do (except for Task 3 ). This was also confirmed by the intensity reported for each task, reflecting from medium to very high intensity (for the disgust tasks). Furthermore, the genuineness distribution rating revealed the spontaneity and genuineness of the emotional expressions displayed by participants. However, as expected and already reported in similar studies (Happy et al. 2015), the elicitation and recording of facial expressions occurring spontaneous emotional experiences is empirically not easy (Tcherkassof et al., 2013). Indeed, the emotional induction varied according to the subjective perception and sensitivity of the participants. For example, Task 1 ("The Lion King") was reported as very sad by most of the subjects, while a few experienced fear or anger. Yet, in Task 11 ("Abused dog in a metro"), most participants revealed to have experienced anger. However, others reported sadness, surprise, or even no emotions (i.e., neutral). Likewise, the intensity of the emotional excitement perceived varied across the tasks and between the subjects (for details of the self-reports of each subject, please see Supplemental Material T3). Importantly, the intensity reported in self reports is not predictive of the emotional expressions shown. For example, even though fear is reported as the second emotion per high level of intensity, the number of the clips is relatively low compared to other emotions (e.g., happiness). Moreover, not all subjects display the entire range of emotions. While happiness and disgust were easy to induce (see Fig. 1), other emotions such as fear and anger were challenging to elicit (possible theoretical interpretations for these results are provided in the section 5).

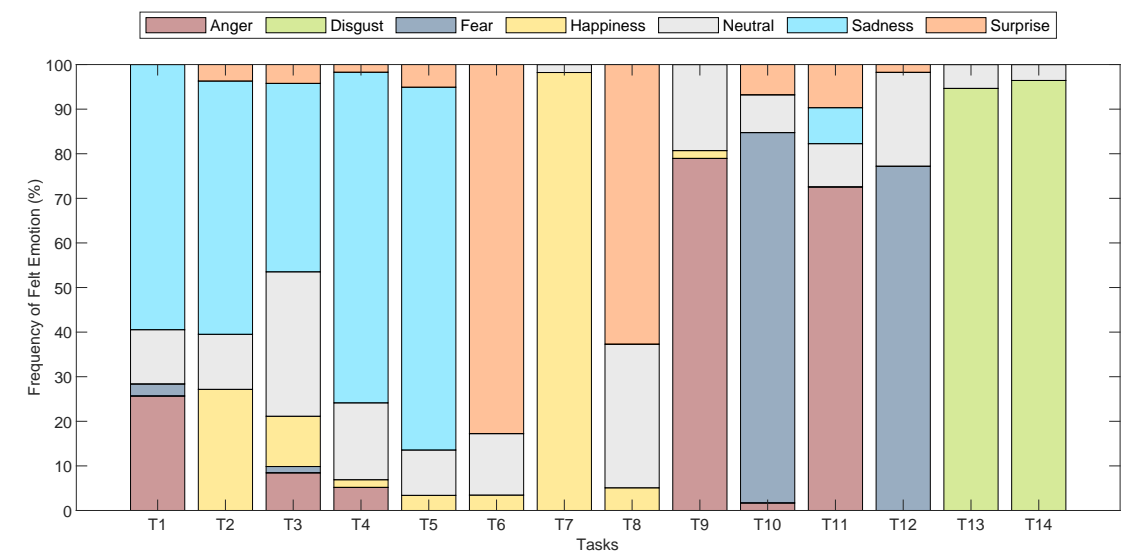

Fig. 3: Emotion distribution from self-report for each task. 


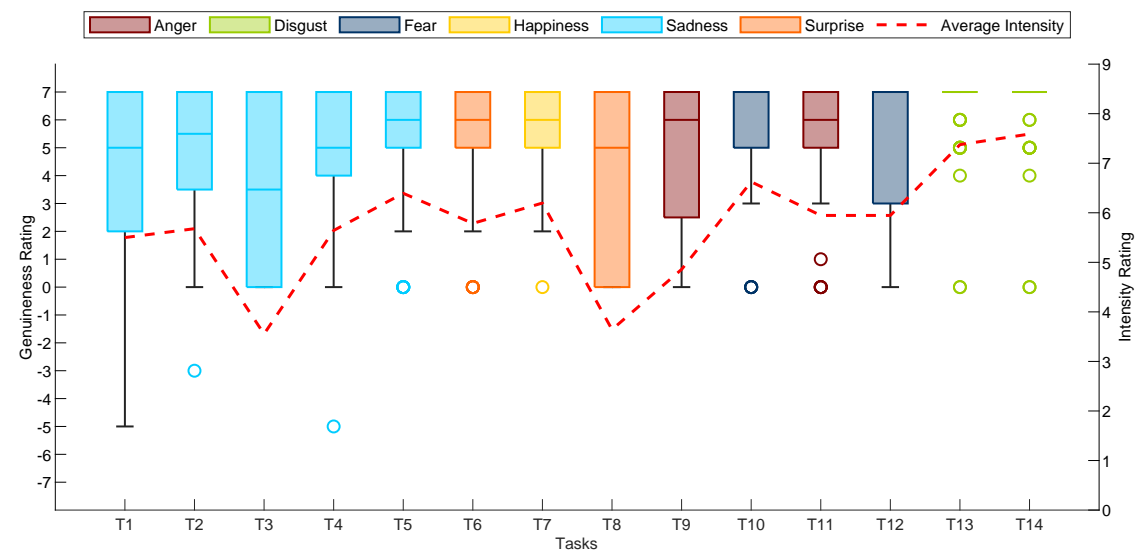

Fig. 4: Genuineness and Intensity rate distribution for each task. The boxplots of participants' ratings are provided for the fourteen elicitation conditions. At the end of each boxplot, the horizontal lines indicate the lower quartile of the responses, while the open circles represent the participants detected as outliers in the boxplot. The red dotted line indicates the average intensity reported in each task.

\section{Validation}

\subsection{Participants}

Being the number of stimuli very high $(N=1731)$, they were split into four independent blocks, each of them including approximately 400 stimuli. Each rater was randomly assigned to one block. A total of 122 participants were recruited for the validation study, matched for age (Mean=25.3; $\mathrm{SD}=2.47)$ and gender (Male $=58 ;$ Female $=64$ ), resulting in each block being validated by 30 independent raters. A further 29 subjects did open the link to the rating task but never started it (i.e., $23.8 \%$ drop-out). Of all 122 participants, 98 (80.3\%) completed the entire rating, while 24 raters $(19.7 \%$ ) did not. Among these, $25 \%$ (6 out of 24 ) completed more than $70 \%$ of the questionnaire. The rest of participants (18 out of 24) partially rated the validation (23.8\% on average), and their data is included. Participants were all graduate students at the University of Padova (Italy). The majority of the participants were recruited through the institute's participant pool. Others were recruited from online University discussion forums.

\subsection{Procedure}

The Validation Procedure was sent online to participants' email addresses using Qualtrics software (http://www.qualtrics.com). Participants were shown short clips displaying facial expressions of anger, disgust, fear, happiness, sadness, and surprise from the PADFE. After each of the emotional clips, participants were asked to categorize the emotion, and the type of expression (i.e., genuine or fake) displayed. Last, participants evaluated how intense the emotions looked at them. The validation was conducted according to Dawel et al.,(2017). The emotion recognition was measured with a fixed-choice question, with all the six presented emo- 
tions plus "neutral" and "none of the above" options (Frank and Stennett, 2001). Thus, participants indicated which emotion label best described the displayed expression. Per the emotion category, we calculated the "hit rates" by dividing the number of accurately recognized emotions by the total number of displays for that emotion. The emotions' genuineness was rated with a 15-points Likert scale, ranging from -7 (completely fake) to 7 (completely genuine). The neutral midpoint "0" corresponded to "I do not know". In other words, all the ratings above "0" indicated a genuine perception of the emotion. Likewise, scores below " 0 " indicated a fake perception of the emotion shown. This method implicates different advantages. First, it allowed us to assess the ratings in absolute terms (i.e., genuine or fake). Second, it provided information regarding the gradient of genuineness perceived by raters (e.g., +7 indicates that the emotion was perceived as genuine without any doubt by the observer, a different gradient from a score of +1 , very close to " 0 "). Doing so, we calculated the "hit rate" of genuineness by dividing the number of accurately recognized emotions as genuine or fake by the total number of displays. Simultaneously, the Mean and the Standard Deviation (SD) of the gradient of genuineness were also calculated. Last, participants rated the intensity perception of the emotion shown on a scale of 0 (none) to 9 (strong). The mean and SD of these parameters were calculated. The questionnaire took about 2 hours and $30 \mathrm{~min}$ to be completed. However, participants were strongly suggested to divide the questionnaire into three days (i.e., 45 minutes of task per day).

\subsection{Validation results}

The "hit rate for emotion" was adopted as the main exclusion criteria for the original 1731 clips. In fact, all the clips recognized with a "hit rate for emotion" less than $30 \%$ were removed from the entire dataset, obtaining 1458 emotional clips (i.e., 707 spontaneous and 751 posed) in total. The list of the final stimuli, including the hit rates for emotion and genuineness, intensity and genuineness rating, as well as the duration of each clip is provided in Supplemental Material T1. In 2 , the total number of clips included in PEDFE, as well as the hit rates, divided for emotion (e.g., disgust) and genuineness (i.e., spontaneous and posed), are reported respectively. Furthermore, the same analysis was conducted more in detail for every single subject actor included in the PADFE's clips (please see Supplemental Material T2). Notably, on average, regardless of genuineness (i.e., spontaneous or posed), all the emotions were categorized with an accuracy of $78.6 \%$, ranging from $58.01 \%$ (for fear) to $93.66 \%$ (for happiness). As expected, happiness is the bestlabeled emotion (both for spontaneous and posed expressions). Conversely, fear is the worst in accordance with the literature that reveal lower recognition rates of fear than the other basic emotions (Roy-Charland et al., 2014). Further analyses were run in order to investigate if the cause of the low accuracy rating of fear was due to the misclassification with the surprise. To do this, we calculate the number of times the emotion was categorized as a surprise for each clip. Results confirmed that, on average, fear is labeled as a surprise $29.76 \%$ of the time (SD 19.71\%). Additionally, to evaluate if the intensity perception of the emotional expressions affects the emotion's discrimination, we conducted the Pearson correlation test. Importantly, the hit rate seems to be moderately affected by the intensity of the 
emotions expressed ( $r=0.44$, for 1458 items), in particular for anger expressions ( $r=0.67$ for 166 items). The correlations between hit rate per emotion and intensity are reported in Supplemental Material T4 for each emotion. For what concerns the hit rate for the genuineness categorization, the global accuracy is stable across all the emotions (i.e., $62.51 \%$ ), ranging from $60.22 \%$ (for disgust) to $65.25 \%$ (for fear). More precisely, genuine emotions were categorized better (i.e., $71.92 \%$ on average) than the posed ones (i.e., $53.65 \%$ on average), regardless of the emotion displayed (please see Fig. 5). Chi-squared test among all the binary responses extract by raters for each emotional stimulus confirmed the significant effect of the type of the stimuli (i.e., spontaneous or posed) on the hit rate of genuineness for each emotion with a $p<0.00001$. In particular, anger $\chi^{2}(1, N=$ $1.4662)=100.65$, disgust $\chi^{2}(1, N=1.7719)=221.97$, fear $\chi^{2}(1, N=1.4049)=$ 164.53 , happiness $\chi^{2}(1, N=1.10876)=376.52$, sadness $\chi^{2}(1, N=1.6619)=$ 172.65 , and surprise $\chi^{2}(1, N=1.5823)=100.94$. In other words, people tended to classify posed emotions as genuine more often than they classify genuine as posed. Differently from the hit rate for emotion, these results are completely unrelated to the intensity $(r=0.11$, for 1458 item) or the emotion $(r=0.06$, for 1458 item) expressed. A theoretical explanation of these results is provided in section 5 .

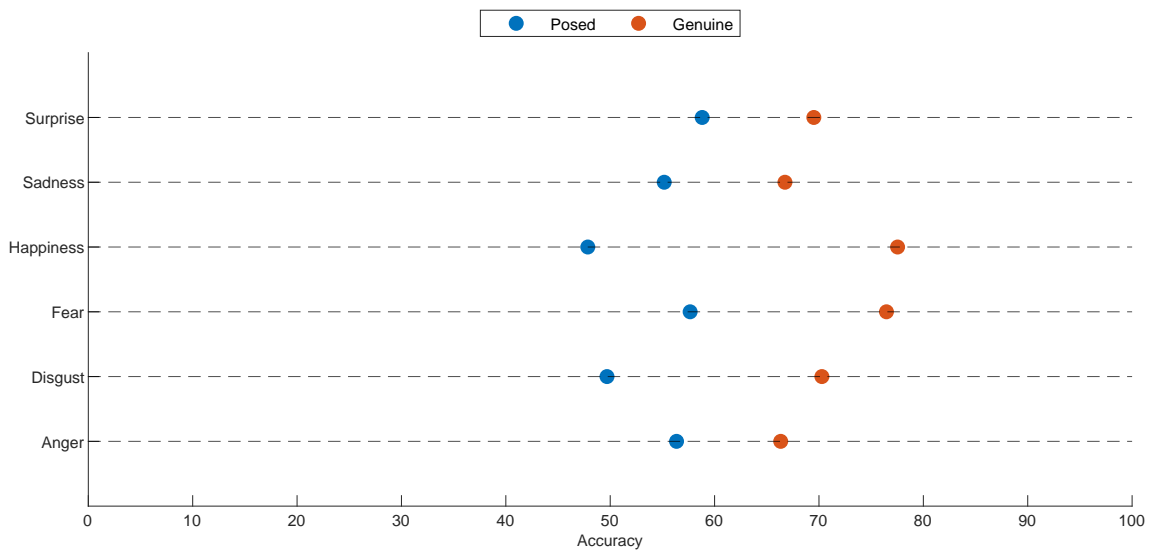

Fig. 5: Genuineness Hit rate for each emotion.

4.4 Video enhancement for Machine Learning applications

After all the emotional facial expressions were rated from the entire validation, the clips were submitted through different video processing steps. These phases aim to obtain clips containing only the face of the participant, removing everything that did not strictly concern facial expression. First, the clips were processed using OpenFace (Baltrušaitis et al., 2016). OpenFace is a face detection software based on deep neural networks that we used to extract for each clip frame containing only the face of the subject (i.e., the background was removed, see Fig. 6). 


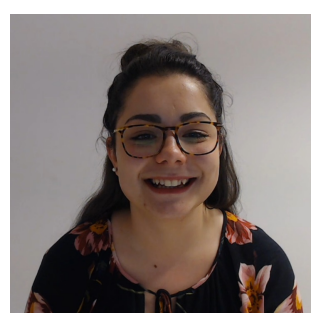

(a) Original clip

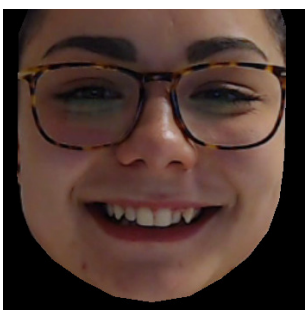

(b) Final clip

Fig. 6: Clip pre and post production

Table 2: Total number of clips included in PEDFE, followed by their respective hit rates.

\begin{tabular}{llllllllll}
\hline & \multirow{2}{*}{ TOT } & \multirow{2}{*}{ POS } & GEN & $\begin{array}{l}\text { HR Emo } \\
\text { TOT (\%) }\end{array}$ & $\begin{array}{l}\text { HR Emo } \\
\text { POS (\%) }\end{array}$ & $\begin{array}{l}\text { HR Emo } \\
\text { GEN (\%) }\end{array}$ & $\begin{array}{l}\text { HR Type } \\
\text { TOT (\%) }\end{array}$ & $\begin{array}{l}\text { HR Type } \\
\text { POS(\%) }\end{array}$ & $\begin{array}{l}\text { HR Type } \\
\text { GEN (\%) }\end{array}$ \\
\hline Anger & 166 & 90 & 76 & 64.88 & 69.30 & 59.64 & 60.92 & 56.36 & 66.33 \\
Disgust & 305 & 149 & 156 & 84.48 & 87.10 & 81.98 & 60.22 & 49.69 & 70.28 \\
Fear & 156 & 93 & 63 & 58.01 & 53.95 & 64.01 & 65.25 & 57.66 & 76.47 \\
Happiness & 370 & 156 & 214 & 93.66 & 93.42 & 93.84 & 65.02 & 47.85 & 77.53 \\
Sadness & 251 & 132 & 119 & 71.09 & 73.57 & 68.35 & 60.66 & 55.18 & 66.74 \\
Surprise & 210 & 131 & 79 & 78.70 & 85.44 & 67.52 & 62.84 & 58.81 & 69.51 \\
\hline ALL & 1458 & 751 & 707 & 78.61 & 79.51 & 77.66 & 62.51 & 53.65 & 71.92
\end{tabular}

Note. TOT: Total number of clips; GEN: Number of Genuine clips; POS: Number of Posed clips; HR Emo TOT: Emotion Hit rate for the total number of clips; HR Emo POS: Emotion Hit rate for Posed clips; HR Emo GEN: Emotion Hit rate for Genuine clips; HR Type TOT: Genuineness Hit rate for the total number of clips; HR Type POS: Genuineness Hit rate for Posed clips; HR Type GEN: Genuineness Hit rate for Genuine clips .

The size of each frame is fixed and was manually set to $300 \times 300$ pixels, meaning that all the extracted faces were resized to fit these constraints. In addition, OpenFace provides bidimensional coordinates of 68 facial landmarks for each frame. To maintain the native dimension of the faces, in order to avoid stretched images, we leveraged the coordinates of the landmarks to resize the frames of each clip. In particular, the maximum difference among $\mathrm{x}$-coordinates and $\mathrm{y}$-coordinates per frame was extracted. We then calculated the median value among all the frames of a clip, obtaining the native size of each face. Finally, we resized each frame of a clip to the corresponding native size, and we padded the frame with black pixels, obtaining new clips of $854 \times 480$ pixels (see Fig. 7). Moreover, for each clip, the pictures captured frame by frame displaying the emotions' temporal dynamics are also provided, except for the clips "5_dg_1" and "30_dg_1" that were successively removed due to the low quality of the recordings. The pictures were included in the dataset available to the scientific community as they can be beneficial to researchers to investigate the course of the emotional expression as well as the various degrees of intensity of the emotions (e.g., from neutral to mid to high intensity) with static pictures. Of note, the kind of emotion expressed by the participant, the genuineness and intensity of emotions felt are obviously not affected by the video enhancement procedure. Researchers who will use these videos should be cautious in generalizing the results of validation to these videos. 


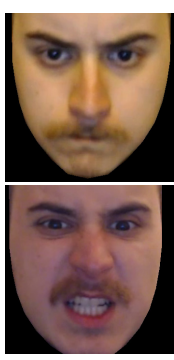

(a) Anger

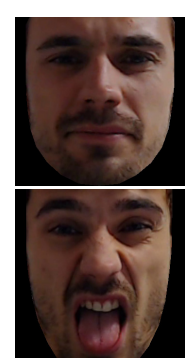

(b) Disgust

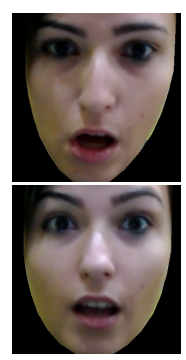

(c) Fear

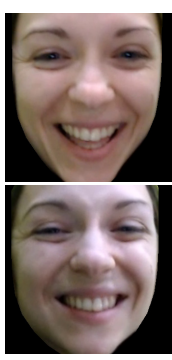

(d) Happiness

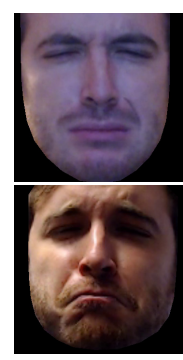

(e) Sadness

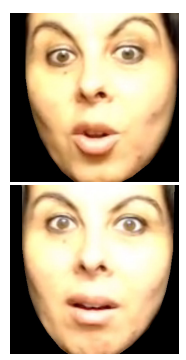

(f) Surprise

Fig. 7: Peak intensity images of genuine (first row) and posed expressions (second row) of the six emotions included in PEDFE.

\section{Discussion}

So far, the emotions conveyed by faces classically used as emotional stimuli in the research on emotions are not genuine. Thus, to date it is still unknown whether our actual knowledge on perception of emotions conveyed by faces is biased by the unconscious perception of the non authenticity of the emotion expressed and thus, if results achieved so far could be generalized to the perception of authentic, more ecological, expressions. The current work aims to provide the scientific community with a new dataset of emotional facial expressions including both spontaneous (i.e. genuine) and posed emotions from the same actor and validated by independent raters. Genuine emotions were elicited using an innovative multi-modal elicitation strategy, that allowed us to select the most effective strategy for each emotion's peculiarity. In the final dataset, which includes 707 spontaneous and 751 posed emotions, facial expressions of the six basic emotions are displayed both in dynamic clips and static pictures. As expected, some emotions such as fear and anger were more challenging to elicit than others (e.g., happiness or disgust) and, as a consequence, the number of stimuli included in the dataset varies according to the emotion expressed. For example, PEDFE contains 370 clips of happiness expressions and "only" 156 of fear and 166 of anger. This finding is perhaps not surprising, considering that fear and anger are known as the most difficult emotions to elicit (Rottenberg et al. 2007). The reason why anger is difficult to elicit might be because anger requires a high level of personal engagement to be experienced (Zupan and Babbage, 2017). The vision of clips and the rage game used in the elicitation protocol might have not triggered high levels of anger in all participants. As with regard of fear, this emotion was in some participants expressed through a passive freezing reaction (Lojowska et al., 2018, Roelofs, 2017), which was translated in a subjective experience of fear in the absence of facial movements. This made the detection and recognition of fear by means of facial clues harder. In addition, stimuli aiming to elicit both anger and fear often cause a blend of negative emotions, such as disgust and sadness in the case of anger, or tension and anxiety in the case of fear (Rottenberg et al., 2007). This likely contribute to the expression of mixed emotions, not surviving to the stringent selection strategy we adopted, consisting in matching the emotion subjectively felt by the participants (rating), with the emotions expressed and codified by a certified FACS expert. This of course contributed to the relatively low number of clips. In general, re- 
gardless of the emotion considered, the collection of spontaneous expressions in an experimental setting is not easy because of a trade-off between ecological reactions and methodological restrictions (Sneddon et al., 2011; Tcherkassof et al. 2013). To make sure that participants' emotional facial expressions were natural and spontaneous, no restrictions (e.g., movements, eye gaze, the intensity of the expressions) were given to participants. This unavoidable compromise made it impossible to match the number of genuine and posed emotions perfectly. Furthermore, the great variability among the participants' sensitivity affected the expressions of emotions both between subjects and within the same subject (i.e., in expressing spontaneous and posed emotions). However, this limitation offers, at the same time, an ecological set of spontaneous facial expressions, providing emotions that differ under different features, such as the intensity of the expression, eye gaze, head movements.

Another contribution comes from the elimination of the background. Indeed, all the incidental features such as hair, clothes, the color of the setting room that may influence emotional expression perception were removed from the background of the stimuli. In other words, only the face on a black screen was portrayed in the clips. A further significant benefit of the isolation of the background concerns the automatic detection of the emotional facial expressions and the face. Indeed, many face recognition algorithms require prior segmentation and alignment or faces, failing with non-uniform background. Isolating the face from the background can help the algorithms align the face to a standard template and improve facial expressions' accurate detection (Tsao and Livingstone, 2008). Future users should however be aware that the independent raters validated the original clips and not the modified ones. However, future users could still benefit from the rater of genuineness and intensity of the felt emotions from the original actors. Notably, all stimuli were validated by human observers. The normative data obtained are in line with the typical finding in expressions databases (Langner et al., 2010, Palermo and Coltheart, 2004). More precisely, the hit rate for emotion is, on average, more than $93 \%$ for happiness and ranging from $64.88 \%$ to $84.48 \%$ for the other emotions. The only exception is fear, where the hit rate for emotion is $58.01 \%$. However, it is noted how fear is easily mistaken for a surprise (Ekman, 1976, Ekman and Friesen, 1971, Rapcsak et al., 2000, Wang and Markham, 1999). The low level of accuracy in fear was indeed due to this typical tendency. In general, the emotion accuracies are moderately correlated with the intensity of the emotion perceived as reported in section Supplemental Material T4. In other words, the more intense the emotion is expressed, the higher is the accuracy rate for the emotion, in accordance with the literature of emotions. It is known how low intensity reduces labeling accuracy, affecting the observers' ability to detect whether or not an expression is shown because of insufficient physical information in the face (Barrett et al., 2019, Dawel et al., 2017, O'Reilly et al., 2016). Different from the hit rate for emotion, the accuracy of the hit rate of genuineness is on average $62.51 \%$, highlighting the inability of humans in (emotional) lie detection. In fact, it is known that people (both untrained observers and professional experts like psychologists) are unable to recognize deceit in emotional displays, in particular, if they have to rely on visual cues only (Bartlett et al. 2006). Several studies demonstrated how people tend to perform not far from the chance level when asked to detect such behaviors (Levine et al., 1999, Porter and Ten Brinke, 2008, Porter and ten Brinke, 2010; Porter et al., 2012; Vrij, 2008). Furthermore, this 
problem is amplified by people's tendency to believe that the person with whom they are speaking is honest, regardless of whether or not that person is lying or being untruthful (Levine, 2014; McCornack and Parks, 1986). This mechanism called truth-bias belongs to human nature to believe and weakens its ability to detect deception. This was also confirmed in the validation of PEDFE, where the hit rate for the genuineness of posed emotion (i.e., when participants should have classified emotions as posed to respond correctly) is on average $53.65 \%$. Conversely, the hit rate for the genuineness of genuine emotion (i.e., when participants should have classified emotions as genuine to respond correctly) is $71.92 \%$. Also note, these results do not change according to the intensity of the emotion expressed. In other words, the intensity of the expression does not improve the accurate detection of spontaneous and posed emotional facial expressions differently for the hit rate for emotion.

\section{Conclusion}

This paper presents a new dataset of facial expressions displaying spontaneous and posed emotions. PEDFE contributes a unique source of ecological stimuli, providing 1458 dynamic clips and the pictures frame by frame of each stimulus. The significant amount of emotions included in PEDFE, offers an excellent choice and a vivid picture of the variability in emotional expressions permeating real-life situations. Furthermore, the normative data give insight into the perception of emotional facial expressions by human observers. PEDFE may be an invaluable resource in different fields of study, such as psychology and analysis of non-verbal behavior, affective computing, and emotional lie detection. Future works will aim to enrich the dataset with new participants and more complex emotions.

\section{Availability}

The PADFE is made freely available to the research community. The Dataset can be obtained by writing a mail at alessio.miolla@phd.unipd.it. An End User License Agreement (EULA) needs to be produced for accessing the database.

\section{Author contributions}

AM and CS conceived the experiment; AM collected and extracted the data; AM and $\mathrm{MC}$ run the data analysis; CS, AM and MC drafted the paper.

\section{Competing interests}

The authors declare no competing interests

\section{Acknowledgments}

The present study received a grant from the University of Padua (Supporting TAlent in ReSearch @ University of Padua - STARS Grants 2017) to CS. 


\section{References}

Ambadar, Z., Schooler, J. W., and Cohn, J. F. (2005). Deciphering the enigmatic face: The importance of facial dynamics in interpreting subtle facial expressions. Psychological science, 16(5):403-410.

Andrew, R. J. (1963). Evolution of facial expression. Science, 142(3595):10341041.

Baltrušaitis, T., Robinson, P., and Morency, L.-P. (2016). Openface: an open source facial behavior analysis toolkit. In 2016 IEEE Winter Conference on Applications of Computer Vision (WACV), pages 1-10. IEEE.

Barrett, L. F., Adolphs, R., Marsella, S., Martinez, A. M., and Pollak, S. D. (2019). Emotional expressions reconsidered: Challenges to inferring emotion from human facial movements. Psychological science in the public interest, 20(1):1-68.

Bartlett, M. S., Littlewort, G., Frank, M. G., Lainscsek, C., Fasel, I. R., Movellan, J. R., et al. (2006). Automatic recognition of facial actions in spontaneous expressions. Journal of multimedia, 1(6):22-35.

Carvalho, S., Leite, J., Galdo-Álvarez, S., and Gonçalves, O. F. (2012). The emotional movie database (emdb): A self-report and psychophysiological study. Applied psychophysiology and biofeedback, 37(4):279-294.

Cheng, S., Kotsia, I., Pantic, M., and Zafeiriou, S. (2018). 4dfab: A large scale 4d database for facial expression analysis and biometric applications. In Proceedings of the IEEE conference on computer vision and pattern recognition, pages 51175126.

Cohn, J. F. and Schmidt, K. (2003). The timing of facial motion in posed and spontaneous smiles. In Active Media Technology, pages 57-69. World Scientific.

Darwin, C. (1872). The expression of emotions in animals and man. London: Murray, 11:1872.

Darwin, C. and Prodger, P. (1998). The expression of the emotions in man and animals. Oxford University Press, USA.

Davidson, R. J., Ekman, P., Saron, C. D., Senulis, J. A., and Friesen, W. V. (1990). Approach-withdrawal and cerebral asymmetry: emotional expression and brain physiology: I. Journal of personality and social psychology, 58(2):330.

Davies, S., Bishop, D., Manstead, A. S., and Tantam, D. (1994). Face perception in children with autism and asperger's syndrome. Journal of Child Psychology and Psychiatry, 35(6):1033-1057.

Dawel, A., Wright, L., Irons, J., Dumbleton, R., Palermo, R., O'Kearney, R., and McKone, E. (2017). Perceived emotion genuineness: normative ratings for popular facial expression stimuli and the development of perceived-as-genuine and perceived-as-fake sets. Behavior Research Methods, 49(4):1539-1562.

Dowell, N. M. and Berman, J. S. (2013). Therapist nonverbal behavior and perceptions of empathy, alliance, and treatment credibility. Journal of Psychotherapy Integration, 23(2):158.

Ekman, P. (1972). Universals and cultural differences in facial expressions of emotion. 1971. URL: https://www. paulekman. com/wpcontent/uploads/2013/07/Universals-And-Cultural-Differences-In-FacialExpressions-Of. pdf (2015-0\%-15).

Ekman, P. (1976). Pictures of facial affect. Consulting Psychologists Press.

Ekman, P., Davidson, R. J., and Friesen, W. V. (1990). The duchenne smile: emotional expression and brain physiology: Ii. Journal of personality and social 
psychology, 58(2):342.

Ekman, P., Friesen, W., and Hager, J. (2002). Facs investigator's guide.(2002).

Ekman, P. and Friesen, W. V. (1971). Constants across cultures in the face and emotion. Journal of personality and social psychology, 17(2):124.

Ekman, P. and Friesen, W. V. (2003). Unmasking the face: A guide to recognizing emotions from facial clues, volume 10. Ishk.

Ekman, P., Friesen, W. V., and Hager, J. (1978). Facial action coding system: manual. palo alto.

Ekman, P. and Rosenberg, E. (2005). What the face reveals. 2nd.

Ekman, P., Sorenson, E. R., and Friesen, W. V. (1969). Pan-cultural elements in facial displays of emotion. Science, 164(3875):86-88.

Ekman, R. (1997). What the face reveals: Basic and applied studies of spontaneous expression using the Facial Action Coding System (FACS). Oxford University Press, USA.

Frank, M. G. and Stennett, J. (2001). The forced-choice paradigm and the perception of facial expressions of emotion. Journal of personality and social psychology, 80(1):75.

Happy, S., Patnaik, P., Routray, A., and Guha, R. (2015). The indian spontaneous expression database for emotion recognition. IEEE Transactions on Affective Computing, 8(1):131-142.

Hayes, C. J., Stevenson, R. J., and Coltheart, M. (2009a). The processing of emotion in patients with huntington's disease: variability and differential deficits in disgust. Cognitive and behavioral neurology, 22(4):249-257.

Hayes, C. J., Stevenson, R. J., and Coltheart, M. (2009b). Production of spontaneous and posed facial expressions in patients with huntington's disease: Impaired communication of disgust. Cognition and Emotion, 23(1):118-134.

Izard, C. E. (1991). The psychology of emotions. Springer Science \& Business Media.

Jack, R. E., Garrod, O. G., Yu, H., Caldara, R., and Schyns, P. G. (2012). Facial expressions of emotion are not culturally universal. Proceedings of the National Academy of Sciences, 109(19):7241-7244.

Jack, R. E. and Schyns, P. G. (2015). The human face as a dynamic tool for social communication. Current Biology, 25(14):R621-R634.

Jack, R. E., Sun, W., Delis, I., Garrod, O. G., and Schyns, P. G. (2016). Four not six: Revealing culturally common facial expressions of emotion. Journal of Experimental Psychology: General, 145(6):708.

Johnston, L., Miles, L., and Macrae, C. N. (2010). Why are you smiling at me? social functions of enjoyment and non-enjoyment smiles. British Journal of Social Psychology, 49(1):107-127.

Jung, E., Wiesjahn, M., Rief, W., and Lincoln, T. M. (2015). Perceived therapist genuineness predicts therapeutic alliance in cognitive behavioural therapy for psychosis. British Journal of Clinical Psychology, 54(1):34-48.

Krumhuber, E. G., Skora, L., Küster, D., and Fou, L. (2017). A review of dynamic datasets for facial expression research. Emotion Review, 9(3):280-292.

Kulkarni, K., Corneanu, C., Ofodile, I., Escalera, S., Baro, X., Hyniewska, S., Allik, J., and Anbarjafari, G. (2018). Automatic recognition of facial displays of unfelt emotions. IEEE transactions on affective computing.

Künecke, J., Wilhelm, O., and Sommer, W. (2017). Emotion recognition in nonverbal face-to-face communication. Journal of Nonverbal Behavior, 41(3):221-238. 
Langner, O., Dotsch, R., Bijlstra, G., Wigboldus, D. H., Hawk, S. T., and Van Knippenberg, A. (2010). Presentation and validation of the radboud faces database. Cognition and emotion, 24(8):1377-1388.

Levine, T. R. (2014). Truth-default theory (tdt) a theory of human deception and deception detection. Journal of Language and Social Psychology, 33(4):378-392.

Levine, T. R., Park, H. S., and McCornack, S. A. (1999). Accuracy in detecting truths and lies: Documenting the "veracity effect". Communications Monographs, 66(2):125-144.

Lojowska, M., Ling, S., Roelofs, K., and Hermans, E. J. (2018). Visuocortical changes during a freezing-like state in humans. Neuroimage, 179:313-325.

Lu, W., Ngai, C. S. B., and Yang, L. (2020). The importance of genuineness in public engagement - an exploratory study of pediatric communication on social media in china. International Journal of Environmental Research and Public Health, 17(19):7078.

Manera, V., Grandi, E., and Colle, L. (2013). Susceptibility to emotional contagion for negative emotions improves detection of smile authenticity. Frontiers in Human Neuroscience, 7:6.

McCornack, S. A. and Parks, M. R. (1986). Deception detection and relationship development: The other side of trust. Annals of the International Communication Association, 9(1):377-389.

Minami, T., Nakajima, K., and Nakauchi, S. (2018). Effects of face and background color on facial expression perception. Frontiers in psychology, 9:1012.

Motley, M. T. and Camden, C. T. (1988). Facial expression of emotion: A comparison of posed expressions versus spontaneous expressions in an interpersonal communication setting. Western Journal of Communication (includes Communication Reports), 52(1):1-22.

Novello, B., Renner, A., Maurer, G., Musse, S., and Arteche, A. (2018). Development of the youth emotion picture set. Perception, 47(10-11):1029-1042.

O'Reilly, H., Pigat, D., Fridenson, S., Berggren, S., Tal, S., Golan, O., Bölte, S., Baron-Cohen, S., and Lundqvist, D. (2016). The eu-emotion stimulus set: a validation study. Behavior research methods, 48(2):567-576.

Palermo, R. and Coltheart, M. (2004). Photographs of facial expression: Accuracy, response times, and ratings of intensity. Behavior Research Methods, Instruments, E Computers, 36(4):634-638.

Porter, S. and Ten Brinke, L. (2008). Reading between the lies: Identifying concealed and falsified emotions in universal facial expressions. Psychological science, 19(5):508-514.

Porter, S. and ten Brinke, L. (2010). The truth about lies: What works in detecting high-stakes deception? Legal and criminological Psychology, 15(1):57-75.

Porter, S., Ten Brinke, L., and Wallace, B. (2012). Secrets and lies: Involuntary leakage in deceptive facial expressions as a function of emotional intensity. Journal of Nonverbal Behavior, 36(1):23-37.

Rapcsak, S. Z., Galper, S., Comer, J., Reminger, S., Nielsen, L., Kaszniak, A., Verfaellie, M., Laguna, J., Labiner, D., and Cohen, R. (2000). Fear recognition deficits after focal brain damage: a cautionary note. Neurology, 54(3):575-575.

Reed, L. I. and DeScioli, P. (2017). The communicative function of sad facial expressions. Evolutionary Psychology, 15(1):1474704917700418.

Roelofs, K. (2017). Freeze for action: neurobiological mechanisms in animal and human freezing. Philosophical Transactions of the Royal Society B: Biological 
Sciences, 372(1718):20160206.

Rooney, B., Benson, C., and Hennessy, E. (2012). The apparent reality of movies and emotional arousal: A study using physiological and self-report measures. Poetics, 40(5):405-422.

Rottenberg, J., Ray, R., Gross, J., Coan, J., and Allen, J. (2007). The handbook of emotion elicitation and assessment. JJB Allen \& JA Coan (Eds.), pages 9-28.

Roy-Charland, A., Perron, M., Beaudry, O., and Eady, K. (2014). Confusion of fear and surprise: A test of the perceptual-attentional limitation hypothesis with eye movement monitoring. Cognition and Emotion, 28(7):1214-1222.

Russell, J. A. (1994). Is there universal recognition of emotion from facial expression? a review of the cross-cultural studies. Psychological bulletin, 115(1):102.

Sato, W. and Yoshikawa, S. (2004). Brief report the dynamic aspects of emotional facial expressions. Cognition and Emotion, 18(5):701-710.

Schnellbacher, J. and Leijssen, M. (2009). The significance of therapist genuineness from the client's perspective. Journal of Humanistic Psychology, 49(2):207-228.

Sebe, N., Lew, M. S., Sun, Y., Cohen, I., Gevers, T., and Huang, T. S. (2007). Authentic facial expression analysis. Image and Vision Computing, 25(12):18561863.

Sneddon, I., McRorie, M., McKeown, G., and Hanratty, J. (2011). The belfast induced natural emotion database. IEEE Transactions on Affective Computing, $3(1): 32-41$.

Soussignan, R. (2002). Duchenne smile, emotional experience, and autonomic reactivity: a test of the facial feedback hypothesis. Emotion, 2(1):52.

Tcherkassof, A., Dupré, D., Meillon, B., Mandran, N., Dubois, M., and Adam, J.M. (2013). Dynemo: A video database of natural facial expressions of emotions. The International Journal of Multimedia $\&$ Its Applications, 5(5):61-80.

Tsao, D. Y. and Livingstone, M. S. (2008). Mechanisms of face perception. Annu. Rev. Neurosci., 31:411-437.

Valstar, M. and Pantic, M. (2010). Induced disgust, happiness and surprise: an addition to the mmi facial expression database. In Proc. 3rd Intern. Workshop on EMOTION (satellite of LREC): Corpora for Research on Emotion and Affect, page 65. Paris, France.

Vergallito, A., Mattavelli, G., Gerfo, E. L., Anzani, S., Rovagnati, V., Speciale, M., Vinai, P., Vinai, P., Vinai, L., and Lauro, L. J. R. (2020). Explicit and implicit responses of seeing own vs. others' emotions: An electromyographic study on the neurophysiological and cognitive basis of the self-mirroring technique. Frontiers in psychology, 11:433.

Vrij, A. (2008). Detecting lies and deceit: Pitfalls and opportunities. John Wiley \& Sons.

Wallbott, H. G. (1990). The relative importance of facial expression and context information in emotion attributions-biases, influence factors, and paradigms. In Advances in psychology, volume 68, pages 275-283. Elsevier.

Wallbott, H. G. and Scherer, K. R. (1986). Cues and channels in emotion recognition. Journal of personality and social psychology, 51(4):690.

Wang, L. and Markham, R. (1999). The development of a series of photographs of chinese facial expressions of emotion. Journal of Cross-Cultural Psychology, 30(4):397-410.

Wehrle, T., Kaiser, S., Schmidt, S., and Scherer, K. R. (2000). Studying the dynamics of emotional expression using synthesized facial muscle movements. 
Journal of personality and social psychology, 78(1):105.

Xu, Q., Yang, Y., Tan, Q., and Zhang, L. (2017). Facial expressions in context: Electrophysiological correlates of the emotional congruency of facial expressions and background scenes. Frontiers in Psychology, 8:2175.

Yoshikawa, S. and Sato, W. (2006). Enhanced perceptual, emotional, and motor processing in response to dynamic facial expressions of emotion 1. Japanese Psychological Research, 48(3):213-222.

Zhang, Z., Girard, J. M., Wu, Y., Zhang, X., Liu, P., Ciftci, U., Canavan, S., Reale, M., Horowitz, A., Yang, H., et al. (2016). Multimodal spontaneous emotion corpus for human behavior analysis. In Proceedings of the IEEE Conference on Computer Vision and Pattern Recognition, pages 3438-3446.

Zloteanu, M., Krumhuber, E. G., and Richardson, D. C. (2018). Detecting genuine and deliberate displays of surprise in static and dynamic faces. Frontiers in Psychology, 9:1184.

Zuckerman, M., Hall, J. A., DeFrank, R. S., and Rosenthal, R. (1976). Encoding and decoding of spontaneous and posed facial expressions. Journal of Personality and Social Psychology, 34(5):966.

Zupan, B. and Babbage, D. R. (2017). Film clips and narrative text as subjective emotion elicitation techniques. The Journal of social psychology, 157(2):194210. 